\title{
SKEWNESS, KURTOSIS AND NEWTON'S INEQUALITY
}

\author{
R. SHARMA AND R. BHANDARI
}

\begin{abstract}
We show that an inequality related to Newton's inequality provides one more relation between skewness and kurtosis. This also gives simple and alternative proofs of the bounds for skewness and kurtosis.
\end{abstract}

1. Introduction. The Pearson [4] inequality:

$$
\alpha_{4} \geq 1+\alpha_{3}^{2}
$$

gives a one-sided relation between skewness and kurtosis, respectively, defined as

$$
\alpha_{3}=\sqrt{\frac{m_{3}^{2}}{m_{2}^{3}}} \quad \text { and } \quad \alpha_{4}=\frac{m_{4}}{m_{2}^{2}},
$$

where

$$
m_{r}=\frac{1}{n} \sum_{i=1}^{n}\left(x_{i}-\bar{x}\right)^{r}, \quad r=1,2, \ldots,
$$

is the $r$ th central moment and $\bar{x}$ is the arithmetic mean of $n$ real numbers $x_{i}(i=1,2, \ldots, n)$. For two different proofs of $(1)$, see $[\mathbf{7}, \mathbf{8}]$.

It is also in statistical interest to bound the sample statistics in terms of the sample size $n$. For example, the [5] inequality states that the standardized maximum deviate $M-\bar{x} / s$ is bounded by $\sqrt{n-1}$, where $M=\max _{i} x_{i}$ and $s=\sqrt{m_{2}}$ is the standard deviation. Wilkins [8] uses the method of Lagrange multipliers to prove that

$$
\left|\alpha_{3}\right| \leq \frac{n-2}{\sqrt{n-1}} .
$$

2010 AMS Mathematics subject classification. Primary 60E15.

Keywords and phrases. Skewness, kurtosis, moments, polynomials.

The authors acknowledge the support of the UGC-SAP.

Received by the editors on December 4, 2013. 
For an alternative proof of (4), see [6]. Dalen [1] proves an upper bound for the kurtosis,

$$
\alpha_{4} \leq \frac{n^{2}-3 n+3}{n-1} .
$$

For a brief history and motivation of these inequalities, see Nicholas [3]. We state here a two-sided relation between skewness and kurtosis and show that the inequalities (4) and (5) follow as a consequence. A refinement of the inequality (5) is given for symmetric distributions.

In an entirely different context, a result due to Newton [2] says that, if all the roots of the polynomial equation

$$
f(x)=a_{0} x^{n}+a_{1} x^{n-1}+\cdots+a_{n}=0
$$

are real, then

$$
a_{i}^{2} \geq \frac{n-i+1}{n-i} \frac{i+1}{i} a_{i+1} a_{i-1}, \quad i=1,2, \ldots, n-1 .
$$

An inequality of our present concern is (7), $i=3$,

$$
a_{3}^{2} \geq \frac{4}{3} \frac{n-2}{n-3} a_{2} a_{4} .
$$

We first prove a refinement of the inequality (8) for the case when the coefficient of $x^{n-1}$ in (6) is zero.

Lemma 1.1. If all the roots of the monic polynomial equation

$$
g(x)=x^{n}+b_{2} x^{n-2}+\cdots+b_{n}=0
$$

are real, then for $n \geq 4$,

$$
b_{3}^{2} \geq \frac{16}{9} \frac{n-2}{n-3} b_{2} b_{4} .
$$

Proof. The $(n-4)$ th derivative of $g(x)$ shows that the roots of polynomial equations,

$$
\text { (11) } \begin{aligned}
n(n-1)(n-2)(n-3) x^{4}+12(n-2)(n-3) b_{2} x^{2} \\
+ \\
+24(n-3) b_{3} x+24 b_{4}=0
\end{aligned}
$$


and

$$
\begin{aligned}
h(y)= & 24 b_{4} y^{4}+24(n-3) b_{3} y^{3}+12(n-2)(n-3) b_{2} y^{2} \\
& +n(n-1)(n-2)(n-3)=0,
\end{aligned}
$$

are all real, $b_{4} \neq 0$. Likewise, it follows on differentiating $h(y)$ that the roots of the equation

$$
4 b_{4} y^{2}+3(n-3) b_{3} y+(n-2)(n-3) b_{2}=0
$$

are real. The inequality (10) now follows from the fact that the discriminant of the quadratic equation (13) is non-negative. For $b_{4}=0$, (10) is obvious.

We now show that the inequality (10) yields an interesting relation between skewness and kurtosis.

Theorem 1.2. Let $\alpha_{3}$ and $\alpha_{4}$ be, respectively, the skewness and kurtosis of $n$ real numbers $x_{1}, x_{2}, \ldots, x_{n}$. Then

$$
1+\alpha_{3}^{2} \leq \alpha_{4} \leq \frac{1}{2} \frac{n-3}{n-2} \alpha_{3}^{2}+\frac{n}{2}, \quad n \geq 3 .
$$

Proof. On using the well-known Newton's identity,

$$
\alpha_{k}+b_{1} \alpha_{k-1}+b_{2} \alpha_{k-2}+\cdots+b_{k-1} \alpha_{1}+k b_{k}=0,
$$

where

$$
\alpha_{k}=\sum_{i=1}^{n} x_{i}^{k}, \quad k=1,2, \ldots n,
$$

we have the following relations between the moments (3) and coefficients $b_{i}$ in polynomial equation (9),

$$
m_{2}=-\frac{2}{n} b_{2}, \quad m_{3}=-\frac{3}{n} b_{3}, \quad m_{4}=\frac{2}{n}\left(b_{2}^{2}-2 b_{4}\right) .
$$

Equivalently,

$$
b_{2}=-\frac{n}{2} m_{2}, \quad b_{3}=-\frac{n}{3} m_{3}, \quad b_{4}=\frac{n^{2}}{8} m_{2}^{2}-\frac{n}{4} m_{4} .
$$

Substituting the values of $b_{2}, b_{3}$ and $b_{4}$ from (15) in (10), a simple calculation leads to second inequality (14). If $b_{4}=0,2 m_{4}=n m_{2}^{2}$ 
and the second inequality (14) is obviously true. The first inequality is (1).

The above theorem provides a complete relation between skewness and kurtosis. The first inequality (14) is Pearson's inequality and the second inequality (14) is its complementary. The inequalities (4) and (5) are subsumed in (14). From (14), we have

$$
1+\alpha_{3}^{2} \leq \frac{1}{2} \frac{n-3}{n-2} \alpha_{3}^{2}+\frac{n}{2} .
$$

Inequality (4) follows easily from (16). Further, the limits of kurtosis in (14) increase with absolute values of skewness. The upper bound for the kurtosis therefore corresponds to the maximum value of $\alpha_{3}$. Substitute $\alpha_{3}=n-2 / \sqrt{n-1}$ in the second inequality (14), a little calculation leads to (5).

If $\alpha_{3}=0$, as in the case of symmetric distribution, a refinement of inequality (5) follows from (14),

$$
\alpha_{4} \leq \frac{n}{2}
$$

\section{REFERENCES}

1. J. Dalen, Algebraic bounds on standardized sample moments, Stat. Prob. Lett. 5 (1987), 329-331.

2. I. Newton, Arithmetica universalis: sive de compositione et resolutione arithmetica liber, 1707.

3. J.C. Nicholas, Speaking stata: The limits of sample skewness and kurtosis, The Stata Journal 10 (2010), 482-495.

4. K. Pearson, Mathematical contributions to the theory of evolution, XIX; Second supplement to a memoir on skew variation, Philos. Trans. Roy. Soc. Lond. 216 (1916), 432.

5. P.A. Samuelson, How deviant can you be?, J. Amer. Stat. Assoc. 63 (1968), $1522-1525$.

6. R. Sharma, R. Bhandari and M. Gupta, Inequalities related to the CauchySchwarz inequality, Sankhya 74 (2012), 101-111.

7. R. Sharma, R. Bhandari and A. Thakur, Some bounds for integrals with refinements of the Gruss inequality, Indian J. Pure Appl. Math. 42 (2011), 187202 .

8. J.E. Wilkins, A note on skewness and kurtosis, Ann. Math. Stat. 15 (1944), $333-335$. 
Department of Mathematics \& Statistics, Himachal Pradesh University, SHIMLA -5, INDiA - 171005

Email address: rajesh_hpu_math@yahoo.co.in

Department of Mathematics \& Statistics, Himachal Pradesh University, SHIMla -5, India - 171005

Email address: raj.maths77@gmail.com 